\title{
«Moderne Zeiten» - Stress, Burnout und Möglichkeiten der Prävention und Intervention
}

\author{
Ein Interview mit Christian Dormann
}

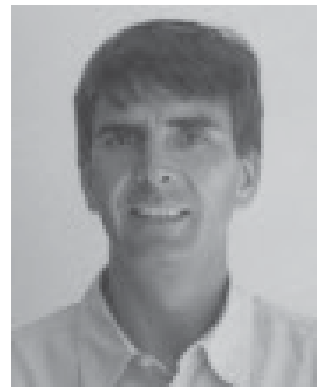

Prof. Dr. Christian Dormann studierte an der Justus-Liebig-Universität Gießen Psychologie und angewandte Informatik. Von 1994 bis 2004 war er als wissenschaftlicher Mitarbeiter und Assistent an den Universitäten Konstanz und Frankfurt/M. tätig. Im Jahr 2000 promovierte er über methodische Probleme der längsschnittlichen Stressforschung, 2004 habilitierte er über psychologische Faktoren in Dienstleistungsinteraktionen. Seit 2004 ist er Leiter der Abteilung für Arbeits-, Organisations-, und Wirtschaftspsychologie der Johannes Gutenberg-Universität Mainz. Seine Forschungsschwerpunkte sind Stress in Organisationen, Dienstleistungen, Arbeitszufriedenheit und evidenzorientiertes Management. Das Gespräch führte Dr. Nadine Schuster, Mainz.

Herr Professor Dormann, die neuesten Gesundheitsreporte bescheinigen uns wieder, dass krankheitsbedingte Fehltage im Arbeitsleben sinken, psychische Erkrankungen jedoch ansteigen. Das Thema Burnout spielt hierbei sicherlich eine große Rolle. Eine holländische Studie besagt, dass 10-15\% der Bevölkerung Burnout-Werte aufweisen, bei denen eine arbeitsplatzbezogene oder medizinische Intervention angesagt wäre. Wie zeigt sich Burnout am Arbeitsplatz und was sind die auslösenden Faktoren?

Christian Dormann: Ursprünglich ist man davon ausgegangen, dass nur Menschen in «People Work», also Menschen in helfenden Berufen, an Burnout erkranken. Es leiden aber auch Menschen in Berufen, die nicht direkt mit Pflege oder Therapie zu tun haben, unter diesen Symptomen. Christina Maslach, die Pionierin im Forschungsfeld Burnout, unterscheidet drei Burnout-Bereiche: Erschöpfung, Depersonalisation/Zynismus sowie reduzierte persönliche Erfüllung. Bei Personen, die nicht in helfenden Berufen arbeiten, ist der beste Prädiktor für den Bereich Erschöpfung ein hoher Zeitdruck bzw. eine hohe Arbeitsbelastung («Work Load»).
Muss also die These, Burnout sei ein Symptom der helfenden Berufe, verworfen werden?

Christian Dormann: Vor 12 Jahren erschien das Buch «The Burnout Companion to Study and Practice» von Wilmar Schaufeli und Dirk Enzmann, die die bis dahin vorliegende Literatur einem Review unterzogen haben. Sie fanden keine Beweise dafür, dass Burnout etwas mit «People Work» zu tun hat. Seit den 1990er Jahren hat sich die Forschung dann konkreter damit auseinandergesetzt, was «People Work» tatsächlich ausmacht. Dabei konnten zwei Faktoren identifiziert werden: Emotionsarbeit und das, was allgemein als «Stress mit Kunden» bezeichnet wird. Stress mit Kunden wird mittlerweile als ebenso wichtiger Prädiktor für die Erschöpfungskomponente des Burnouts angesehen wie Zeitdruck. Unter dem Aspekt «Stress mit Kunden» werden unter anderem Verbalaggressionen, Unfreundlichkeit und Unschlüssigkeit von Kunden zusammengefasst werden. Auch für die zweite Burnout-Komponente, den Zynismus bzw. die Entfremdung, ist «Stress mit Kunden» der beste Prädiktor. Es ist jedoch mittlerweile klar, dass es bei der Entfremdungskomponente nicht nur um den Stress mit Kunden geht, sondern ganz allgemein um Stress in sozialen Beziehungen bei der Arbeit.

\begin{tabular}{ll}
\hline KARGER & @ 2010 S. Karger GmbH, Freiburg \\
Fax +497614520714 & Accessible online at: \\
Information@Karger.de & www.karger.com/ver \\
www.karger.com &
\end{tabular}

Prof. Dr. Christian Dormann

Abteilung Arbeits-, Organisations- und Wirtschaftspsychologie

Psychologisches Institut der Johannes Gutenberg-Universität Mainz

Wallstr. 3, 55122 Mainz, Deutschland

Tel. +49 6131 39-39246, Fax -39247

cdormann@uni-mainz.de 
Christian Dormann: Der zentrale Aspekt im Konzept der Emotionsarbeit ist die sogenannte «emotionale Dissonanz». Damit ist gemeint, dass der Betroffene gezwungen ist, eine bestimmte Emotion zu zeigen, obgleich sie nicht der real empfundenen entspricht. Dies kann entweder von der Organisation erfordert oder selbst auferlegt sein. Die sogenannten «Display Rules», d.h. Verhaltensregeln, bestimmen das Ausmaß dieser Dissonanz. Auch hier steht der soziale Aspekt im Mittelpunkt. Zentral ist das «Surface Acting», also das Vorspielen von Emotionen, weil es sozial erwünscht ist. Im Gegensatz zum «Deep Acting», also dem Nachvollziehen der Emotionen des Gegenübers, ist dieses Vorspielen von Emotionen ein «Burnout-Treiber», wie er sonst seinesgleichen sucht. Surface Acting und Deep Acting werden in der letzten Zeit verstärkt auch im Zusammenhang mit Betrieben und gewünschtem Verhalten am Arbeitsplatz untersucht. Firmen erlegen sich selbst emotionsorientierte Verhaltenskodizes («Immer lächeln») auf, die bei den Mitarbeitern häufig zu emotionaler Dissonanz und zu chronischer Emotionsregulationsüberforderung führt.

Wir erleben ja heute eine Renaissance der Selbstständigkeit. Der SPIEGEL-Artikel «Moderne Zeiten» (SPIEGEL 12/2010) verweist auf eine sich verändernde gesellschaftliche und arbeitsmarktbezogene Struktur, die sich seit einigen Jahren unaufhaltsam zeigt: Die Realität sieht heute so aus, dass weniger als zwei Drittel der Erwerbstätigen einer sozialversicherungspflichtigen Tätigkeit nachgehen. Wie wirkt sich das auf das Erleben und Verhalten der Beschäftigten aus?

Ein immer größer werdender Teil der arbeitenden Bevölkerung arbeitet in der Selbstständigkeit, jeder Auftraggeber ist ein Kunde. Stress mit Kunden bzw. Emotionsarbeit werden daher immer wichtiger. Sie können sich vorstellen, dass der soziale Aspekt bei Selbstständigen Grundlage des Geschäftes ist. Hier können die Prädiktoren für Burnout also besonders stark wirken. Auch Leiharbeit spielt eine Rolle. Leiharbeit war früher vor allem bei geringer qualifizierten Tätigkeiten verbreitet. Mittlerweile ist Leiharbeit jedoch auch häufig der erste bezahlte Job bei jungen Menschen, die gerade ihr Hochschulstudium abgeschlossen haben. Emotionsmanagement spielt dabei eine herausragende Rolle und muss als eigenständig zu erlernende Fertigkeit angesehen werden. Emotionsmanagement ist deshalb so wichtig, weil man als Leiharbeiter ständig mit neuen Leuten zu tun hat, sich ständig auf neue Kunden und neue Chefs einstellen muss und weil man für den Leiharbeitgeber eine wichtige Arbeitskraft bleiben möchte. Spannend ist jedoch, dass die psychische Belastung der Leiharbeiter geringer ist, als vermutet werden könnte. Im Gegenteil: Forschungsergebnisse zeigen, dass es den Festangestellten in einer Firma mit vielen Leiharbeitern psychisch schlechter geht als den Mitarbeitern einer Firma mit wenigen Leiharbeitern.
Christian Dormann: Die hohe Präsenz von Leiharbeitern übt Druck auf die Festangestellten aus. Angst ist die vorherrschende Emotion in Unternehmen mit vielen Leiharbeitern: Man sieht, dass Arbeitsverhältnisse unsicherer werden. Es wird deutlich, dass Kollegen leicht ersetzbar sind. Dadurch entstehen auch Bedenken hinsichtlich der Sicherheit des eigenen Arbeitsplatzes. Das gleiche gilt für sogenannte «multiple layoffs», also wiederholte Entlassungswellen. Früher galt die Hypothese, dass durch das «Überleben» wiederholter Entlassungswellen Copingfähigkeiten ausgebildet werden, die uns in die Lage versetzen, mit solch bedrohlichen Situationen umzugehen. Diese Annahme hat sich jedoch nicht bewahrheitet. Es geht den Mitarbeitern umso schlechter, je mehr Entlassungswellen sie durchlaufen haben. Die ständige Unsicherheit und der ständige Druck überwiegen die Entwicklung von Copingfähigkeiten bei Weitem.

\section{Die Angst, der Nächste zu sein, wird größer?}

Christian Dormann: Ja, genau. Zu Anfang hat man immer gesagt, dass eine gewisse Routine entsteht, nach dem Motto: «Jetzt habe ich schon die vierte Welle überlebt, und ich bin immer noch da. So bedrohlich ist es dann auch nicht.» Aber das Gegenteil ist der Fall.

Wie, glauben Sie, wird die Entwicklung in den nächsten Jahren sein? Wo liegen die Belastungen im Arbeitsleben?

Christian Dormann: Lange Zeit standen die körperlichen Belastungen im Vordergrund. Danach standen die eher kognitiven Belastungen im Mittelpunkt, also Zeitdruck, Konzentration etc., darauf folgen heute die emotionalen Belastungen. Was wir in Zukunft stärker vorfinden werden, sind die motivationalen Belastungen. Dies hat etwas mit der Renaissance der Selbstständigkeit zu tun, aber auch damit, dass die Umwelten immer dynamischer werden, dass den Vorgesetzten immer häufiger «the facts of the case» fehlen, dass die Mitarbeiter oft mehr Wissen haben als ihre Vorgesetzten und deswegen mehr Handlungsfreiräume zugebilligt bekommen müssen. Mit der Weitergabe von Handlungsspielräumen an die Mitarbeiter hinken wir in Deutschland weit hinter anderen Ländern Westeuropas hinterher. Die Mitarbeiter werden in Deutschland zunehmend mehr Verantwortung und Handlungsspielräume bekommen. Die Universität ist ein Beispiel: Jeder Student, der von einer stark strukturierten Schule an die Uni kommt, kennt das - er hat auf einmal viele Freiheiten. Und wer es nicht schafft, damit umzugehen, der fällt raus. Und bei den zunehmenden Anforderungen an die Selbstständigkeit, wird das immer relevanter - auch in Betrieben. Wenn Mitarbeiter also nicht entsprechend qualifiziert sind und nicht gelernt haben, Selbstmanagement - die Grundlage für selbstständiges Handeln - zu betreiben, ent- 
steht mäßiger Stress. Es sammeln sich vielleicht immer mehr Aufträge an, die noch nicht ausgeführt sind, und die Leute verfallen in eine Art Schreckstarre, nach dem Motto: Das ist so viel, das schaffe ich nicht, ich mache erst gar nicht daran weiter. Diese motivationalen Belastungen werden zunehmen. Die Einführung der Bachelorstudiengänge dürfte dabei eher ungünstig wirken.

Sie meinen, dass die Einführung der neuen Studienabschlüsse einen Einfluss hat auf die Selbstmanagementfähigkeiten von Studierenden?

Christian Dormann: Ja. In vielen Studiengängen, gerade in solchen wie Soziologie oder Erziehungswissenschaften, haben sich Studenten früher ihren Weg durch die Universität mit viel Eigenenergie gesucht. Sie haben versucht, mit den Unwägbarkeiten und Unsicherheiten klarzukommen. Es gab keine Sanktionsmaßnahmen, wenn man lange studiert hat. Man hat nur etwas gemacht, wenn man sich selbst positioniert oder motiviert hat. Wer hingegen heute studiert, der wird primär durch externe Kontrolle strukturiert. Das ist zwar im Sinne der formalen Abschlüsse gut. Dadurch gehen aber Stressbewältigungs- und dadurch faktisch Selbstmanagementkompetenzen verloren. Alles wird elektronisch bereitgestellt, keiner wühlt sich mehr durch und weiß mehr, wie man sich die Informationen beschafft, die man braucht - was eben an der Universität auch wichtig ist. Wir sind eine Informationsgesellschaft - in der muss man sich Informationen besorgen. Das heißt, viele Kompetenzen, die man traditionell vermittelt hat, gehen heute verloren. Das ist allerdings noch Zukunftsmusik, Studien hierzu liegen noch nicht vor, es wird aber spannend sein, diese Entwicklung $\mathrm{zu}$ beobachten und $\mathrm{zu}$ begleiten.

Herr Dormann, welches wissenschaftliche Modell ist aktuell vorherrschend zur Erklärung von Burnout?

Christian Dormann: Das dominierende Modell in der Literatur ist sicherlich das Job Demands-Resources(JD-R)-Modell von Arnold Bakker und Kollegen. Dem Modell liegt die Annahme zugrunde, dass jede Arbeitstätigkeit eigene, spezifische Ursachen für Mitarbeitergesundheit beinhaltet. Diese Faktoren werden unterteilt in zwei generelle Kategorien: Arbeitsanforderungen und Ressourcen. Sie liefern ein übergreifendes Modell für verschiedene Beschäftigungssettings, unabhängig von den spezifischen Anforderungen und Ressourcen einer Tätigkeit. Es gibt also zwei Prozesse, die simultan stattfinden: Hohe Arbeitsanforderungen erschöpfen die mentalen und körperlichen Ressourcen der Mitarbeiter und führen zu Energieverlust und gesundheitlichen Problemen; dieser Prozess wird als «health erosion process», also gesundheitsschädigender Prozess bezeichnet. Im Gegensatz dazu fördern arbeitsbezogene Ressourcen das Mitarbeiterengagement und das Extra-Rollenverhalten ...
... also Verhaltensweisen von Mitarbeitern am Arbeitsplatz, die freiwillig gezeigt werden, nicht explizit im Arbeitsvertrag geregelt sind und positive oder negative Auswirkungen auf die Effektivität der Organisation haben.

Christian Dormann: Genau. Dieser Prozess wird als «motivational process» bezeichnet. Arbeitsplatzbezogene Ressourcen können den Einfluss von Arbeitsanforderungen auf die individuelle Stressreaktion abpuffern. Außerdem können arbeitsplatzbezogene Ressourcen auch ein enormes Motivationspotenzial haben, wenn hohe Arbeitsanforderungen vorliegen. Arbeitsbezogene Ressourcen sind beispielsweise Handlungsspielraum, soziale Unterstützung durch den Vorgesetzten oder Unterstützung durch die Kollegen.

\section{Wie entwickelt sich Burnout nach diesem Modell?}

Christian Dormann: Hier entwickelt sich Burnout im Wesentlichen dadurch, dass man auf der Arbeit sehr vielen Stressoren ausgesetzt ist: Stress mit Kunden, Zeitdruck etc. Dieser Pfad wird «Stresspfad» genannt. Engagement hingegen entwickelt sich eher, wenn beispielweise ein angemessener Handlungsspielraum und soziale Unterstützung von Kollegen oder Vorgesetzten, sprich hohe Ressourcen, verfügbar sind oder - was wir auch gerade untersuchen - soziale Unterstützung vonseiten des Kunden hinzukommt. Man spricht hier vom «Engagement-Pfad». Johannes Siegrist verdeutlicht die Bedeutung von Gratifikationen, also Anerkennungen für geleistete Arbeit. Gratifikationen müssen nicht materieller Art sein, sondern reichen von Wertschätzung von Vorgesetzten oder internen und externen Kunden bis zu Weiterbildungsangeboten und familienfreundlichen Strukturen. Diese Ressourcen haben einen Einfluss auf das Engagement der Mitarbeiter. «Je mehr soziale Unterstützung, desto höher mein Engagement ...», und können Burnout vorbeugen.

Gibt es denn auch Situationen, in denen ein großer Handlungsspielraum negative Auswirkungen haben kann?

Christian Dormann: Stellen Sie sich eine Zeit vor, in der große Arbeitsplatzunsicherheit herrscht. Mitarbeiter können denken: «Es ist ohnehin gerade alles so unsicher, und jetzt muss ich auch noch selbstständig Entscheidungen treffen.» Manche Menschen haben so etwas wie ein «desire for control», also den Wunsch, Handlungsspielräume zu haben; anderen ist es lieber, wenn sie gesagt bekommen, was sie zu tun haben. Dies beeinflusst auch, ob sich Handlungsspielraum positiv oder negativ auf einen Mitarbeiter auswirkt. Forschungsergebnisse aus den 1970er Jahren besagen, dass Mitglieder teilautonomer Arbeitsgruppen, die zu Beginn eines Projektes keinen Wunsch nach Handlungsspielraum verspürten, nach Abschluss des Projektes dieses gerade auch aufgrund der eigenen Kontrolle sehr 
positiv bewerteten. Menschen wollen unter Umständen zunächst keine Handlungsspielräume haben und keine Verantwortung übernehmen. Wenn sie damit positive Erfahrungen machen, kann dies jedoch sehr motivierend wirken.

Mitarbeiterorientierte Führung würde hierbei bedeuten, dass ich jedem Mitarbeiter das gebe, was er oder sie braucht.

Christian Dormann: Das ist richtig. Nehmen wir beispielsweise das situative Führungsmodell von Hersey und Blanchard, das die vier Führungsstile Unterweisen bzw. Anweisen («telling»), Verkaufen («selling»), Beteiligen («participating») und Delegieren («delegating») unterscheidet. Als Situationsvariablen werden die Fähigkeit der Mitarbeiter bezüglich der zu realisierenden Aufgabe, d.h. das Maß an Fachwissen, Fertigkeiten und Erfahrung, sowie die Bereitschaft bzw. Motivation zur Aufgabenrealisierung einbezogen. Ausgehend vom Entwicklungsstand des Mitarbeiters wird dann der geeignete Führungsstil bestimmt. «Unreife» Mitarbeiter müssen in diesem Modell eher an die Hand genommen werden; mit steigendem Reifegrad des Mitarbeiters lässt sich die Aufgabenorientierung reduzieren und die Beziehungsorientierung verstärken.

Neben den Faktoren in der Arbeitswelt gibt es sicherlich auch psychische Dispositionen, die Burnout eher entstehen lassen. Welche Faktoren in der Person selbst tragen Ihres Wissens nach dazu bei, dass Arbeit krank macht?

Christian Dormann: In der Person selbst tragen Feindseligkeit, Typ-A-Verhalten, aber vor allem Neurotizismus dazu bei, dass Arbeitsstress entsteht. Neurotizismus ist aus drei unterschiedlichen Gründen problematisch. Erstens, neurotische Personen bzw. Personen mit hohem negativem Affekt nehmen dieselben Stressoren intensiver wahr als Personen ohne dieses Persönlichkeitsmerkmal. Zweitens kann Neurotizismus auch dazu führen, dass Menschen tatsächlich mehr Stress haben als andere. Sie haben einen Selektionsnachteil bei der Personalauswahl, d.h. je höher der Neurotizismus desto geringer die Wahrscheinlichkeit, für einen Job genommen zu werden. Solche Personen müssen sich öfter bewerben und erhalten eher die weniger beliebten Jobs, also diejenigen Jobs, bei denen sie grundsätzlich mehr negativen Stressoren ausgesetzt sind. Neurotizismus kann drittens auch dazu führen, dass man über weniger Ressourcen verfügt, wenn man sich z.B. als hochängstlicher Mensch weniger soziale Unterstützung holt. Für Extraversion finden wir gegenteilige Befunde, also eher einen positiven Selektionsvorteil, mehr positive soziale Kontakte und eine positive Grundhaltung. Dies sind die wesentlichen Faktoren in der Person, die diskutiert werden.
Welche Möglichkeiten haben nun der Einzelne und der Betrieb, sowohl primär-als auch sekundärpräventiv? Wie müsste ein gutes betriebliches Gesundheitsmanagement aufgestell sein?

Christian Dormann: In Deutschland glauben zu wenige Personen, dass Stress wirklich krank macht. Nur die Engländer sind noch weniger davon überzeugt, dass der Job die Gesundheit beeinflusst. Deutschland liegt in dieser Frage weit unter dem europäischen Durchschnitt. Es ist also gar nicht so leicht, über diese Frage in Deutschland zu diskutieren. Des Weiteren schauen die Personen viel zu einseitig nur auf die Stressoren, die man in vielen Fällen gar nicht reduzieren kann. Kunden zum Beispiel, als Ursache von Stress, sind sehr schwer erziehbar. Außerdem legen vor allem in Deutschland Vorgesetzte viel zu häufig ihren Fokus auf Aufgabenorientierung und Kontrolle von Arbeitsergebnissen und sind viel zu wenig auf Mitarbeiterförderung ausgerichtet. Hier lässt sich sicherlich gut ansetzen. Stressorenreduktion würde demnach bedeuten, dass der Betrieb Führungsleitlinien erarbeitet, die der Führungskraft kommuniziert und die trainiert werden müssen. Die sogenannten Soft Skills einer Führungskraft sind hier relevant. Dies ist das eine. Aber worüber viel zu wenig nachgedacht wird, ist der Bereich, wie Ressourcen für den Mitarbeiter aufgebaut werden können. Auch hier sind die Führungskräfte in der Verantwortung, da sie diejenigen sind, die Handlungsspielräume einräumen, die soziale Unterstützung bieten. Sie üben eine Human-Resources-Funktion aus. Sie müssen sich über die Entwicklung ihrer Mitarbeiter Gedanken machen - und darauf müssen die Führungskräfte vorbereitet werden. Vorgesetzte sind die wesentliche Schaltstelle zum primärpräventiven Abbau von Stressoren und zum Aufbau von Ressourcen dort, wo es möglich ist. Soziale Unterstützung durch den Vorgesetzten ist, das zeigt die Forschung, viel wichtiger als soziale Unterstützung durch die Kollegen.

Die Führungskraft hat die Aufgabe, Handlungsspielräume zu gewähren. Wie sieht denn ein konkretes Beispiel zur Steigerung von Handlungsspielraum aus?

Christian Dormann: Nehmen wir an, dass es in einer Abteilung häufig stressbedingtes Fehlen gibt. Ein erster Schritt wäre festzustellen, was die Beschäftigten als ursächlich für ihre Belastung angeben. Stellt sich heraus, dass es fehlender Handlungsspielraum ist, dann müsste zunächst überprüft werden, ob tatsächlich keine Spielräume vorhanden sind oder ob die Mitarbeiter vorhandene Spielräume nicht wahrnehmen. Dann müsste in Mitarbeitergesprächen erfragt werden, wo sich die Mitarbeiter konkret mehr Handlungsspielraum wünschen. Mit den Vorgesetzten muss über ihre Rolle als Führungskraft gesprochen werden und darüber, welche Handlungsspielräume den Mitarbeitern gegeben werden können. Das Vorgehen wäre dann folgendes, dass Mitarbeitern für kleinere Projekte Verantwortung übertragen würde und 
sie hierdurch an mehr Handlungsspielraum herangeführt würden. Menschen wachsen an ihren Aufgaben.

Auf Arbeitnehmerseite selbst steht natürlich die proaktive Steigerung der eigenen Kompetenzen und Fähigkeiten im Vordergrund («Employability»), was zum einen Stressoren abbaut, zum anderen aber auch Handlungsspielräume qua Qualifikation erweitert. Meines Erachtens greifen Stressbewältigungstrainings hier zu kurz.

Als «Employee Assistance Program» (EAP) bezeichnet man im Personalwesen Programme zur (externen) Mitarbeiterberatung. Während in Deutschland bislang nur wenige Konzerne explizit derartige Programme aufgelegt haben, gehört EAP in den USA zum Unternehmensalltag zahlreicher Firmen. Was halten Sie davon?

Christian Dormann: Ursprünglich entwickelt wurden diese Programme in den 1930er Jahren, um Beschäftigte mit Suchtproblemen zu unterstützen. Heutzutage dient ein EAP dazu, Mitarbeiter bei beruflichen und privaten Problemen qualifiziert zu beraten. Wichtige Bereiche sind die Beratung bei psychischen Belastungen am Arbeitsplatz, Suchtberatung, Konfliktberatung, Beratung bei organisatorischen Problemen (z.B. Kinderbetreuung, Pflege von Angehörigen) sowie Beratung in Bezug auf die Work-Life-Balance. Die Inanspruchnahme eines EAP vonseiten des Mitarbeiters kann eine niederschwellige Eingangspforte sein, überhaupt Beratungsbedarf in Anspruch zu nehmen. In den USA gibt es das Modell schon lange, es rechnet sich sehr, und die Mitarbeiter profitieren davon.

In Ihrer Abteilung forscht Frau Professor Binnewies zum Thema Erholung. Auch dies spielt in diesem Zusammenhang eine große Rolle. Gibt es Befunde darüber, wann die Freizeitgestaltung erholsam ist?

Christian Dormann: Ein Hauptergebnis ist, dass es nicht darauf ankommt, was man macht, sondern wie man das, was man tut, erlebt. Man kann sich auch beim Fernsehen erholen, wenn man das, was man sich anschaut, positiv bewertet, z.B.: «Ich habe etwas gelernt dabei.» Gute Erholung wirkt sich direkt auf das Verhalten am Arbeitsplatz aus. Sind Mitarbeiter besser erholt, zeigen sie eine bessere Aufgabenleistung, mehr Eigeninitiative und mehr Hilfeverhalten gegenüber ihren Kollegen. Für die Erholtheit wiederum ist guter Schlaf, das Abschalten und Entspannen von der Arbeit in der Freizeit sowie das Meistern von Herausforderungen (z.B. beim Sport oder bei ehrenamtlichen Tätigkeiten) förderlich. Die Forschungsergebnisse zeigen, dass das morgendliche Befinden der Stu- dienteilnehmer davon abhing, was sie abends in ihrer Freizeit gemacht und wie gut sie geschlafen hatten. Teilnehmer, bei denen die Gedanken abends noch um die Arbeit kreisten, fühlten sich am nächsten Tag müde und schlecht. Am Feierabend von der Arbeit abzuschalten und sich einem Hobby, der Familie und Freunden zu widmen, kann helfen, bewusst eine klare Grenze zwischen Arbeit und Freizeit zu ziehen.

Ist es wichtig, dass ich mich bewusst für eine bestimmte Freizeittätigkeit entscheide, damit sie erholsam ist?

Christian Dormann: Das ist eine interessante Frage. Ich würde sagen, dass es nicht nötig ist, darüber zu reflektieren, sondern dass die Intensität des Erlebten eine Rolle spielt, dass man erlebt, wie die Anspannung aus dem Körper weicht. Nur wenn man das erlebt, ist es erholsam. Dies setzt allerdings eine Reflexion darüber, was man machen kann, was Spaß oder Herausforderung bringen könnte, voraus. Erholung lässt sich - genauso wie Work-Life-Balance - erlernen, ich muss mich intensiv damit auseinandersetzen. Die Forschung bei Top-Managern zeigt, dass diejenigen, die die klarste Grenze zwischen Arbeit und Freizeit ziehen, am erfolgreichsten sind und das erfüllteste Freizeitleben haben. Führungskräfte leben das auch vor.

Arbeit macht glücklich und gesund. Arbeit macht krank. - Was stimmt denn nun?

Christian Dormann: Erst mal ist es ganz wichtig festzuhalten, dass keine Arbeit zu haben, krank macht, und dass Nichtarbeiten keinesfalls glücklich macht. Eine hohe Anzahl von positiven Erlebnissen wird heutzutage in der Arbeit gewonnen. Flow-Erlebnisse, bei denen man an seine geistig-kognitiven Grenzen stößt, passieren häufiger in der Arbeit als in der Freizeit. Es kommt nun einfach darauf an, wie sie gestaltet ist. Die überwiegende Mehrzahl der Personen profitiert davon, zur Arbeit zu gehen. Fragt man Menschen, was sie bei einem Lottogewinn tun würden, sagen viele, dass sie ihre Tätigkeit aufgeben würden. Studien an echten Lottogewinnern zeigen, dass im Schnitt tatsächlich nur 10-12\% der Menschen aufhören zu arbeiten. Menschen gehen gerne arbeiten, denn Arbeit gibt mehr als nur Geld. Hacker spricht von der «Fortsetzung des kindlichen Spiels in der Arbeit». Arbeit muss jedoch gesundheitserhaltend oder gesundheitsförderlich sein, und hierbei spielt der Aufbau von betrieblichen und individuellen Ressourcen zum Umgang mit Belastungen eine entscheidende Rolle.

Herr Professor Dormann, ich danke Ihnen für dieses Gespräch. 